East African Medical Journal Vol. 85 No. 3 March 2008

PLAQUE AND GROWTHCHARACTERISTICS OF DIFFERENT POLIOVIRUSES ISOLATED FROM ACUTE FLACCID PARALYSIS IN NORTHERN NIGERIA

W. F. Sule, DVM, MSc, Lecturer, O. I. Oyedele, PhD, Lecturer, Department of Virology, College of Medicine, University of Ibadan, Nigeria and The Health Systems Trust, 49 Jorrison, Street, Braamfontein, Johannesburg, South Africa, M. OseiKwasi, PhD, Senior Research Fellow, J. K. Odoom, BSc, MSc., Principal Research Assistant, Department of Virology, Noguchi Memorial Institute of Medical Research, College of Health Sciences, University of Ghana, Accra, Ghana and F. D. Adu, DVM, PhD, Professor, Department of Virology, College of Medicine, University of Ibadan, Nigeria

Request for reprints to: Prof. F. D. Adu, Department of Virology, College of Medicine, University of Ibadan, Nigeria

\title{
PLAQUE AND GROWTH CHARACTERISTICS OF DIFFERENT POLIOVIRUSES ISOLATED FROM ACUTE FLACCID PARALYSIS IN NORTHERN NIGERIA
}

\author{
W. F. SULE, O. I. OYEDELE, M. OSEI-KWASI, J. K. ODOOM and F. D. ADU
}

\begin{abstract}
Objective: To determine some virulent trait-related properties of poliovirus isolates from children with acute flaccid paralysis following vaccination with oral polio vaccine (OPV).

Design: Six polioviruses earlier characterised into wild, vaccine-derived and OPV-like were studied using the plaque morphology and growth kinetics at supra-optimal temperature.

Setting: Department of Virology, University of Ibadan, Nigeria.

Subjects: Polio isolates from six children who developed acute flaccid paralysis following vaccinations with various doses of OPV were used. All the children were located in the Northern part of the country where poliovirus is still circulating.

Main outcome measures: The two vaccine-derived polioviruses acquired wild type characteristics.

Results: All the six poliovirus isolates developed different forms of plaques ranging from tiny, small and large. The plaque formed could however not be used to identify the different isolates. Growth of the different isolates at supra-optimal temperature showed that the three wild polioviruses grew to a higher titre when compared with the Sabin 2 control. The two vaccine derived isolates behaved like the wild poliovirus while the OPV-like virus acquired an intermediate characteristics between wild and sabin.

Conclusion: The wild polioviruses represented in this study are among the last vestiges of the circulating polioviruses found in the world. It is possible that the observed biological properties of wild types 1 and 3 described in the study are typical of the West African polioviruses. These properties will provide useful previews to the final identification of some important clinical isolates especially type 1 which may grow rapidly in cell culture.
\end{abstract}

\section{INTRODUCTION}

When poliovirus is confined to the gut, it is harmless, otherwise, it may induce paralysis following viraemia and CNS invasion (1). This is usually the case for wild poliovirus infection. However some live-attenuated vaccine polioviruses that have re-acquired wild-like properties following replication in human gut also show neurovirulence like the wild strains (2) and are excreted by oral polio vaccine (OPV) recipients (3).

Nigeria, which remains one of the last four poliovirus endemic nations, uses the live-attenuated trivalent OPV in routine vaccination of children and in Supplementary Immunisation Activities (SIAs). This has resulted in elimination of several genetic lineages of wild polioviruses in Nigeria though the 
OPV coverage has not been high enough to get rid of the last reservoirs of the virus. Globally, as the circulation of wild polioviruses is decreasing, vaccinederived polioviruses,(VDPV) as well as, OPV-like viruses associated with paralytic poliomyelitis, although rare, are causing increasing concern.

Poliovirus is among the most rapidly evolving viruses known, with about one percent nucleotide substitution per site per year (4). Therefore, OPV recipients usually excrete altered vaccine viruses after vaccination (3). Acquired alterations in the base sequence of the altered vaccine viruses may change their degree of attenuation (5) as well as, their temperature sensitivity(ts) (2). All the three Sabin strains are temperature-sensitive, producing lower virus yields at supra-optimal temperatures than the wild poliovirus (6). Loss of attenuation (3) and temperature sensitivity (7) by derivatives of OPV strains excreted by OPV recipients differentiate them from their corresponding attenuated and temperature sensitive Sabin virus strains (2). The development of techniques for growing polioviruses in tissue culture allowed the recognition of certain characteristics of clonal virus population that can serve as markers for attenuation and as differentiation between wild-type and attenuated Sabin vaccine viruses (8).

Plaque morphology of viruses had been correlated with virulence (9) while temperature sensitivity and plaque formation in tissue culture have been used to determine the degree of attenuation and neurovirulence of poliovirus isolates (7). This study was carried out to determine some biological and plaque properties of these selected polio viruses which were isolated from acute flaccid paralysis patients vaccinated with the oral polio vaccine(OPV).

\section{MATERIALS AND METHODS}

The details of the subjects and the polio viruses used in the study are provided in Table 1.
Viruses: The six polioviruses used in this study were categorised into three groups: wild type, vaccine derived and OPV- like sabin. All the viruses were isolated from the stool of children who developed various degrees of acute flaccid paralysis following vaccination with various doses of the OPV. Virus REC2/ 0455 was initially detected as a SL like virus by ELISA but was found to be an abnormal Sabin using the REC primers developed by Kilpatrick et al (10). Sequence result showed a two nucleotide changes in the VP1 but a vaccine/non-vaccine recombinant in the 3D region (11). Virus VD2/0119 was a VDPV with 22 nucleotide changes in the VPI capsid region and HEV-C enterovirus combination in the 5'-UTR and 3D non-capsid region (12). Isolates WPI/898, WPI/007 and WP3/230 were identified as wild poliovirus types 1 and 3 respectively while isolate OPV2/ 098 was identified as a sabin-like polio virus. Viruses were initially isolated and identified as polio viruses at the WHONational Polio Laboratory, Ibadan according to WHO recommended procedures (13) and intra-typically differentiated as earlier described $(9,20)$. The intratypic typing ( ITD ) which identified the isolates as either Sabin, or wild- type was done at the WHO Regional Laboratory, Ghana using the ELISA method, with cross-adsorbed type-specific polyclonal antibodies (14) while the sequencing was done at the WHO special Polio Laboratory at the Centres for Disease Control, Atlanta, USA, using Sabin-specific primers $(15,16)$. The isolates were recovered from the Ibadan laboratory for the present study.

Reference Sabin-2 strain: This control virus was the live-attenuated Sabin vaccine poliovirus type 2 (Sabin-2) from National Institute of Biological Standard and Control (NIBSC), London, England, with reference number $01 / 530$. This reference Sabin2 strain had been stored at $-20^{\circ} \mathrm{C}$.

Table 1

History of poliovirus isolates

\begin{tabular}{|c|c|c|c|c|c|c|c|c|}
\hline & & & & Ssolat & $\mathrm{d}$ from & & & \\
\hline$S / N$ & J Virus ID & Lab ID & $\begin{array}{l}\text { Age } \\
\text { (Month }\end{array}$ & $\begin{array}{l}\text { Sex } \\
\text { s) }\end{array}$ & Location & Vac. history & Type & $\begin{array}{l}\text { Results of } 60 \\
\text { day follow-up }\end{array}$ \\
\hline 1. & 3D REC2 / 0455 & IBD / $02 / 0455$ & 21 & $\mathrm{M}$ & Plateau & 3 doses of OPV & 2 & NPC \\
\hline 2 & VD2/0119 & IBD / $01 / 0119$ & 16 & M & Bauchi & 2 doses of OPV & 2 & PC \\
\hline 3. & WP1/ 898 & IBD / 03 / 899 & 12 & $\mathrm{~F}$ & Kebbi & 4 doses of OPV & 1 & PC \\
\hline 4. & WP1/007 & IBD / 02/ 007 & 23 & $\mathrm{M}$ & Kaduna & 2 doses of OPV & 1 & PC \\
\hline 5. & WP3/230 & $\mathrm{IBD} / 02 / 230$ & 12 & $\mathrm{~F}$ & Abuja & 5 doses of OPV & 3 & PC \\
\hline 6. & OPV2/098 & IBD / $02 / 098$ & 16 & $\mathrm{~F}$ & Kwara & 3 doses of OPV & 2 & PC \\
\hline
\end{tabular}

$\mathrm{ID}=$ Identity, $\mathrm{NPC}=$ Non - Polio Compatible, $\mathrm{PC}=$ Polio Compatible, $\mathrm{VD}=$ Vaccine - derived, $\mathrm{WP}=\mathrm{Wild}$ poliovirus, $\mathrm{OPV}=$ Oral Polio Vaccine-like virus, $3 \mathrm{D}$ Rec $=3 \mathrm{D}$ Recombinant 
Cells: The three cell lines used in this study were HEp-2C (human larynx carcinoma), L20B (murine L cells expressing human poliovirus receptor), and RD (human rhabdomyosarcoma) cell lines. All the cells were supplied by WHO to the polio laboratory network.

Virus stock: Preparations of poliovirus stocks were done according to standard procedure for virological investigation of poliovirus (18). Viruses were passaged in monolayers of L20B cells and RD cells to produce high-titre viruses for this study. Each virus stock was stored at $-20^{\circ} \mathrm{C}$.

Plaque assays: Plaque assay was done in HEp-2C cell monolayers at $37^{\circ} \mathrm{C}$ as described by Burleson et al (17).

Phenotypic study of plaques and determination of plaque titres: The plaque morphology was visually observed and scored for such characteristics like size; nature of plaque centre and the nature of plaque edges. Plaque size was determined by measuring the cross-diameter of each discrete plaque with a 30 centimetre-rule. Plaque sizes were recorded in millimetres ( $\mathrm{mm}$ ). Plaque titre for each poliovirus isolate was calculated as described by Burleson et al (17).

One-step growth curves: The growth rates of all the isolates at $39.5^{\circ} \mathrm{C}$ in HEp-2C cells were compared with that of Sabin-2 virus in one-step growth experiments at a multiplicity of infection of five plaque-forming units / cell in tissue culture tubes as described by Burleson, et al (17). Titres of virus samples were determined by $50 \%$. Tissue Culture Infective Dose $\left(\mathrm{TCID}_{50} / \mathrm{ml}\right)$ in $\mathrm{L} 20 \mathrm{~B}$ cells incubated at $37^{\circ} \mathrm{C}$ as described earlier (17).

\section{RESULTS}

Virus stock: All the viruses grew to appreciable plaqueforming unit titres in HEp-2C cells after passaging in L20B and RD cell lines. Titres ranged between $6.3 \mathrm{x}$ $10^{6}$ to $2.1 \times 10^{8}$. These titres were used for the plaque and the growth characteristics.

Plaque characteristics of the virus isolates: The plaque characteristics of the poliovirus isolates are as shown in Table 2. The average plaque diameter ranged between $0.9 \mathrm{~mm}$ and $3.6 \mathrm{~mm}$. Plaque morphologies were of various types- tiny, small and large with either clear centres or serrated edges.

One-step growth curves of the isolates: The one-step growth curve is shown in Figure 1. The wild polio viruses grew to higher titres (range Log 10 $0^{5.8}$ - Log $10^{9.3}$ ) when compared with the Sabin 2 control with titre range between $\log 10^{4.8}$ to $\log 10^{7.8}$ after eight hours at $39.5^{\circ} \mathrm{c}$. The two vaccine derived isolates behaved in their growth at $39.5^{\circ} \mathrm{clike}$ the wild isolates while the OPV isolate acquired a growth characteristic that was intermediate between the wild and the Sabin 2 control.

Figure 1

One-step growth curve of polioviruses isolated from acute flaccid paralysis cases

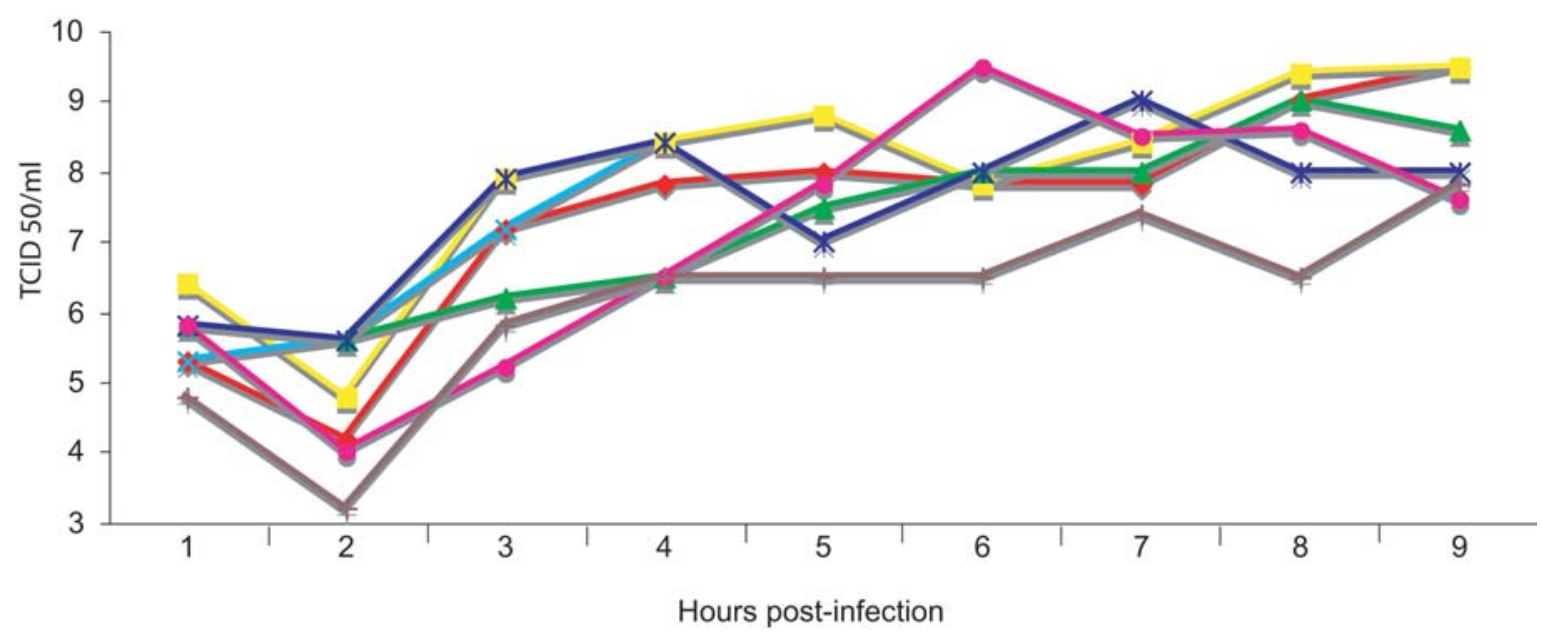

$\longrightarrow$ VD2/045 - VD2/011 $\longrightarrow$ WP1/898 $\rightarrow$ WP3/230 $\rightarrow$ WP1/007 $\longrightarrow$ OPV2/09 $\longrightarrow$ SABIN-2 
Table 2

Plaque titres and morphology of polioviruses at $37^{\circ} \mathrm{C}$ in HEp-2C cell monolayer

\begin{tabular}{|c|c|c|c|}
\hline Poliovirus & $\begin{array}{l}\text { Average HEp-2C } \\
\text { plaque titre } \\
(\mathrm{PFU} / \mathrm{ml})\end{array}$ & $\begin{array}{l}\text { Average plaque } \\
\text { diameter }(\mathrm{mm})\end{array}$ & Other plaque features \\
\hline 3D Rec/0455 & $3.4 \times 10^{7}$ & 1.9 & $\begin{array}{l}\text { Mixture of tiny and small plaques with clear centre } \\
\text { and serrated edges }\end{array}$ \\
\hline VD2/0119 & $6.3 \times 10^{6}$ & 1.1 & Tiny plaques with clear centres \\
\hline WP1/ 899 & $2.1 \times 10^{8}$ & 3.6 & Large plaques with serrated edges and centre \\
\hline WP1/ 007 & $1.3 \times 10^{8}$ & 3.6 & Large plaques with serrated edges and centre \\
\hline WP3/ 230 & $1.4 \times 10^{7}$ & 1.0 & Tiny plaques with clear centre \\
\hline OPV2 / 098 & $3.3 \times 10^{7}$ & 0.9 & Tiny plaques with clear centre \\
\hline Sabin 2 & $7.5 \times 10^{6}$ & 1.1 & Tiny plaques with clear centre \\
\hline
\end{tabular}

\section{DISCUSSION}

As part of her effort to stop the transmission of the circulating wild poliovirus, Nigeria had embarked on mass and intensive use of the oral polio vaccine (OPV) during National Immunisation Days (NIDs) as well as during Sub-National Immunisation Days(SNIDs). However wild poliovirus has continued to circulate in Nigeria. Many factors, of which low population immunity had been identified, is responsible for the continous circulation of these wild polioviruses and rapid evolution and spread of revertant polioviruses (3). In this study we had attempted to determine some phenotypic virulent traits that must have been responsible for the behaviour of some wild poliovirus and revertant isolates by characterising their plaque and growth kinetics.

Assessment of the temperature sensitivities of all the wild-type isolates revealed that they were heat-stable at $39.5^{\circ} \mathrm{C}$ in $\mathrm{HEp}-2 \mathrm{C}$ cell line unlike the reference Sabin-2 virus. Two of the isolates -VD2/ 0455 and VD2/0119 which had some significant changes in their nucleotide sequences(11,12) were similar to the wild viruses in their insensitivity to the elevated temperature. They all grew rapidly to high titres right from zero to eight hour period while the referenceSabin 2 strain exhibited a marked sensitivity to this elevated temperature (Figure 1). This is in agreement with the findings of Yang, et al. (2) who observed some degree of loss of temperature sensitivity for some vaccine-derived polioviruses from polio cases in Egypt. The behaviour of these two isolates is an indication of some loss of some degree of attenuation during replication as evidenced by the fact that two substitutions associated with the attenuated phenotype had either recombined out $\left(\mathrm{A}_{481} \rightarrow \mathrm{G}\right.$ in the $\left.5^{\prime}-\mathrm{UTR}\right)$ or reverted ( $\mathrm{Ile}_{143} \rightarrow$ Thr in VPI) in both these two isolates(12).

When changes in the OPV viruses resulted in re-acquisition of transmissibility and neurovirulence, like their wild counterparts $(2,18)$ they may induce paralytic disease called vaccine-associated paralytic poliomyelitis (VAPP) in OPV recipients and in their susceptible contacts (19). Such vaccine poliovirus strains have been reported as the cause of VAPP outbreaks in different places(20).

The current reinvestigation of the growth properties of different poliovirus isolates may have broader applicability than the earlier studies because the wild polioviruses represented in this study are among the last vestiges of the circulating polio viruses found in the world. It is therefore possible that the observed biological properties of the wild type 1 and 3 isolates described in Table 2 are typical of the West African polioviruses. These properties will definitely provide some useful previews to the final identification of some important clinical isolates especially for type 1 wild polioviruses, which may grow rapidly in cell culture. It is also possible that the more vigorous growth of the wild type 1 polioviruses when compared to the wild type 3 polioviruses could account partly for the wider geographical distribution of wild type 1 poliovirus. It was also interesting that the divergent type 2 vaccine-derived poliovirus grew better than Sabin 2 or the other type 2 vaccines related polioviruses in this study.

The OPV-like isolate showed a similarity in its temperature sensitivity to the Sabin 2 virus only in the first three hours of replication at $39.5^{\circ} \mathrm{C}$, after which it lost temperature sensitivity and behaved like the wild and the vaccine- derived isolates. This behaviour might be unconnected with its association with the AFP case which was later confirmed to be polio compatible by the 60-day follow-up. In contrast to the findings of Yang, et al (2) who reported that OPV-like isolate was temperature sensitive at $39.5^{\circ} \mathrm{C}$ in BGM and HeLa cells respectively, the reason for loss of temperature sensitivity of OPV2/098 observed in this study may be due to some mutational changes in the sequence. It will therefore be interesting to sequence this isolate for further understanding. 
Both the VD2/0119 and VD/0455 exhibited two types of plaque morphology. While VD2/0119 exhibited tiny plaques similar to the WP3/230 wild isolate, the VD2/ 0455 showed a mixture of small and tiny plaques. The behaviours of these two viruses in their plaque morphology may be unconnected with changes they sustained in their genomes, the VD2/ 0119 had three nucleotide changes in its VP1 region, while VD2 / 0455 had 22 nucleotide changes in its VP1 region $(11,12)$. The case with which VD2/ 0119 was associated was non-polio compatible as there was no residual paralysis. Though the VD2/0119 vaccinederived virus actually evolved in the course of replication in GIT of the vaccinee, it could besuggested that it did not evolve towards neuropathogenicity of the wild type, as once observed by Georgescu, et al (4) and Martin, et al (19). This might be the reason for the non-polio compatibility of the case. Also, since none of these two viruses showed large plaques, it may be suggested that the mutations they sustained only influence temperature sensitivity, but did not enhance their replication competence. The different polioviruses irrespective of their plaque phenotypes exhibited different degrees of paralysis in the patients.

\section{ACKNOWLEDGEMENTS}

To the various state Surveillance Officers who sent the samples from the affected States. We also acknowledge the different laboratories which contributed towards isolation and typing of the various isolates. We thank Mr. Rasaq Agunbiade, Mrs. Funke Oyero and Mr. Yinka Abidoye who retrieved the viruses for this study. The assistance of Mr. Seun Adedeji and Dr. Bola Oyemakinde who were responsible for providing the cell lines is immensely appreciated.

\section{REFERENCES}

1. Minor, P. D. Poliovirus vaccination: Current understanding of poliovirus interactions in human and implications for eradication of poliomyelitis . Expert Review Molec. Med. 1999; 23: 1-17.

2. Yang, C.F., Naguib, T., Yang, S.J., et al. Endemic circulation of type 2 vaccine- derived poliovirus in Egypt. 1983 to 1993. J. Virol. 2003; 77: 15-8366.

3. Alexander, J.P., Jr., Gray and H.E., Jr. and Pallanseh, M.A. Duration of poliovirus excretion and its importance for acute flaccid paralysis surveillance: A review of the literature J. Infect. Dis. 1997; 175: (Suppl.1), 5176-5182.

4. Martin, J., Dunn, G., Hull R., et al. Evolution of Sabin strain of type 3 poliovirus in an immunodefficient patient during the entire 637-day period of virus exrection. J. Virol. 2000. 74: 3001-3010.

5. Minor, P.D., The molecular biology of polio vaccines. J. Gen. Virol. 1992; 73: 3065-3077.

6. Bouehard, M.J., Lam, D.A. and Raeaniello, V.R. Determinants of attenuation and temperative sensitivity in the type 1 poliovirus sabin vaccine. J.Virol. 1995; 169: 4972-4978.

7. Nakano, J., Hateh, M., Thieme, M., et al. Parameters for differentiating vaccine-derived and wild poliovirus strains. Progr. Med. Virol. 1978; 24: 178-206.

8. Omata, T., Kohara, M., Kuge, S., et al. Genetic analysis of the attenuation phenotype of poliovirus types. J.Virol. 1986; 58: 348-358.

9. Adu, F.D., Oyejide, A. and Tomori O. 1solation of a clone of low virulence from a Nigerian velogenic strain of New castle disease virus. Off. Int. Epiz. 7: 631-634.

10. Kilpatrick, D.R., Ching, K., Iber, et al. Multiplex PCR method for identifying recombinant vaccine-related poliovirus. J. Clin. Microbiol. 2004; 42: 4315-4319.

11. Adu, F.D., Iber, J., Harry, T., et al. Some genetic characteristics of Sabin-like poliovirus isolated from acute flaccid paralysis cases in Nigeria. Africa J. Biotech. 2003; 2: 460-464.

12. Adu, F.D., Iber, Jane, et al. Isolation of recombinant type 2 vaccine-derived poliovirus (VDPV) from a Nigerian child. Virus Res. 2007; 127: 17-25 .

13. World Health Organisation. WHO/EPI/GEN/97.01. EPI: 1997 Manual for the virological investigation of polio. World Health Organisation, Geneva.

14. Van der Avoort, H.G., Hull, B.P., Hovi, T., R. et al. Comparative study of five methods for intratypic differentiation of polioviruses. J. Clin. Microbiol. 1995; 33: 2562-2566.

15. Kilpatrick, D.R., Nottay, B., Yang, C.F., et al.Serotypespecific identification of polioviruses by PCR using primers containing mixed-base or deoxyinosine residue at position of codon degeneracy. J. Clin. Microbio. 1998; 36: 352-357.

16. Yang, C.F., De, L., Holloway, B.P., Pallansch, et al. Detection and identification of vaccine-related polioviruses by polymerase chain reaction. Virus Res. 1991: 20: 159-179.

17. Burleson, F.G., Chambers, T.M. and Wieldbrauk, D.L. Plaque Assays. In: Virology, A Laboratory Manual, 1992. Academic Press. p 75.

18. Kew, O., Morris-Glasgow, Landerverde, U., et al. Outbreak of poliomyelitis in Hispaniola associated with circulating type 1 vaccine-derived poliovirus. Science. 2002; 296: 356-359.

19. Georgescu, M.M., Balanant, J., Macadam, A., et al. Evolution of Sabin type 1 poliovirus in humans: characterization of strains isolated from patients with vaccine-associated paralytic poliomyelitis. J. Virol. 71: 7758-7768.

20. John, T.J., Vaccine-associated paralytic polio in India. Bull. WHO. 2002; 80: 917. 